\title{
25 Research Soure \\ Clinical Diagnostic Value for Colorectal cancer Based on Serum CEA, CA24-2 and CA19-9
}

Hui Rao

Meizhou People's Hospital

Heming Wu

Meizhou People's Hospital

Qingyan Huang

Meizhou People's Hospital

Zhikang Yu

Meizhou People's Hospital

Qunji Zhang

Meizhou People's Hospital

Zhixiong Zhong ( $\backsim$ zhongzhixiong@mzrmyy.com )

Meizhou People's Hospital

Research

Keywords: Colorectal cancer, Tumor markers, Clinical value, Combined detection

Posted Date: March 27th, 2020

DOl: https://doi.org/10.21203/rs.3.rs-19403/v1

License: @ (i) This work is licensed under a Creative Commons Attribution 4.0 International License. Read Full License 


\section{Abstract}

Background To explore the clinical value of a combined detection of serum concentration of carcinoembryonic antigen (CEA), carbohydrate antigen 24-2 (CA24-2), and carbohydrate antigen 19-9 (CA19-9) for colorectal cancer (CRC).

Methods The levels of serum tumor markers (CEA, CA24-2 and CA19-9) and clinicopathologic features in patients with colorectal cancer in Meizhou People's Hospital were evaluated. In addition, KRAS/NRAS, PIK3CA and BRAF mutations were detected in some patients with colorectal cancer.

Results A total of 2,281 patients (1,420 males and 861 females) were recruited in the study, included 1,578 colorectal cancer patients and 703 controls. The levels of CEA, CA24-2 and CA19-9 in colorectal cancer group was significantly higher than control group. The sensitivities of three individual markers were lower than $30 \%$, which individual sensitivity of the tumor markers sorted in descending order were CEA>CA19-9> CA24-2. The specificities of three individual markers were more than $92 \%$, and the specificity sorted in descending order were CA24-2> CA19-9> CEA. The prediction equation excluding the risk of colorectal cancer was. Probability (normal) $=\operatorname{Exp}(-5.47-0.28 C E A-0.11$ CA242 + 0.001 CA199)/ (1+ Exp (-5.47 - 0.28CEA - 0.11 CA242 + 0.001 CA199)). There were no significant differences in age, gender, histology type, differentiation, depth of invasion and TNM stage between mutations in KRAS/NRAS, BRAF and PIK3CA genes or not, respectively.

Conclusions Serum CEA, ca24-2, and ca19-9 are valuable noninvasive indicators for the detection and screening of patients with early colon cancer. We need to look for other, more sensitive tumor markers.

\section{Introduction}

Malignant tumor diseases occurring in the mucosal epithelium and glands of the rectum and other parts of the colon, collectively referred to as colorectal cancer, colorectal cancer by sigmoid colon and rectal junction is more common[1, 2]. Colorectal cancer (CRC) is one of the most common malignant tumors in clinical practice[3, 4]. Because the early symptoms of the patients are not obvious enough, most of the patients were already in the advanced stage when they were admitted to the hospital, which seriously threatened the life of the patients[5-9]. Therefore, the rapid and correct diagnosis of colorectal cancer is helpful for clinical treatment, so as to improve the efficacy of patients.

In recent years, the detection of serum tumor markers has played an important role in the diagnosis of colorectal cancer and the assessment of the disease due to its high efficiency and non-invasive characteristics, and has been widely used in clinical practice[10, 11]. At present, CEA and CAI9-9 are tumor markers that are widely used in clinical practice. Due to the low mono-positive rate in colorectal cancer, their clinical application value is limited. In addition, CA24-2 can be used for the diagnosis of colorectal cancer[1214]. Some studies have shown that the combined detection of tumor markers can improve the detection rate of malignant tumors[1517], but there are few studies on the combined detection of CEA, CA19-9, CA24-2 and CA72-4 in the diagnosis of colorectal cancer and the relationship between CEA, CA19-9, CA24-2 and CA72-4 and clinical TNM staging.

There has been no systematic study on colorectal cancer patients and tumor markers in Hakka population. In this study, the data of colorectal cancer patients were analyzed retrospectively, who had detected tumor markers from January 2016 to May 2019, also analyzed the data of patients who had detected KRAS/NRAS, BRAF and ALK/ROS1 gene, in order to explore the value of a combined application of serum tumor markers in clinical diagnosis for colorectal cancer.

\section{Materials And Methods}

\section{Participants}

This retrospective clinical study included 1,578 patients with colorectal cancer and 703 controls who visited Meizhou People's Hospital (Huangtang Hospital), Meizhou Academy of Medical Sciences, Meizhou Hospital Affiliated to Sun Yat-sen University between January 2016 and May 2019. This study was conducted on the basis of the Declaration of Helsinki and was supported by the Ethics Committee of the Meizhou People's Hospital.

\section{Sample preparation and tumor markers detection}


Three milliliters of blood samples were obtained from each subject. CEA, CA19-9 and CA24-2 were measured routinely by flow fluorescence method with Quantitative Detection Kit for Tumor Markers (Tellgen Life Science, Shanghai, China). Critical value of CEA, CA19-9 and CA24-2 was $5.00 \mathrm{ng} / \mathrm{mL}, 37.00 \mathrm{U} / \mathrm{mL}$ and $20.00 \mathrm{U} / \mathrm{mL}$. The results were considered positive when any of the markers were positive, and negative when all of the markers were negative.

\section{DNA extraction}

Ten slices of formalin-fixed and paraffin-embedded (FFPE) slices ( $5 \mu \mathrm{m}$ thick per slice) were dewaxed in xylene and rehydrated in descending grades of ethanol. DNA was extracted by AmoyDx ${ }^{\circledR}$ Tissue DNA Kit (Spin Column) (Amoy Diagnostics, Xiamen, China), following the manufacturers' instructions and NanoDrop 2000 Spectrophotometer (Thermo Fisher Scientific, Waltham, MA) was used to evaluate the quantity and quality of extracted DNA.

\section{Amplification-refractory mutation system-polymerase chain reaction (ARMS-PCR) for KRAS/NRAS, PIK3CA and BRAF mutations}

We tested mutations in the KRAS (exons 2, 3 and 4), NRAS (exons 2, 3 and 4), PIK3CA (exon 9 and 20) and BRAF (exon 15) genes using Human KRAS/NRAS Mutations Detection Kit, Human PIK3CA Five Mutations Detection Kit and Human BRAF Mutation Detection Kit (Amoy Diagnostics Co. Ltd, Xiamen, China), respectively. PCR Amplifications were performed with initial denaturation at $95^{\circ} \mathrm{C}$ for 5 minnutes, followed by 15 cycles of first amplification (at $95^{\circ} \mathrm{C}$ for 25 seconds, $64^{\circ} \mathrm{C}$ for 20 seconds and $72^{\circ} \mathrm{C}$ for 20 seconds) and 31 cycles of second amplification (at $95^{\circ} \mathrm{C}$ for 25 seconds, $60^{\circ} \mathrm{C}$ for 35 seconds and $72^{\circ} \mathrm{C}$ for 20 seconds) in the LightCycler 480 real-time PCR system (Roche Diagnostics, Germany). Positive results were defined as $\mathrm{Ct}$ (sample)-Ct (control) < Ct (cut-off). These molecular arrays allow to identify the most important mutations of $K R A S$ (codons $12,13,61,117$, and 146), NRAS (codons 12, 61, 117, and 146), BRAF (codons 600), and PIK3CA genes (codons 542, 545 and 1047).

\section{Statistical analysis}

SPSS statistical software version 21.0 was used for data analysis. The levels of tumor markers were expressed as the means \pm SD. The rank sum test was used to compare the levels of tumor markers in each group and the chi-square $\left(x^{2}\right)$ test was used to compare the rates or constituent ratios. To compare the diagnostic accuracy of detection markers in predicting pathological type of colorectal cancer, the receiver operator characteristic (ROC) curves were generated and the area under the curve was calculated. A value of $P<0.05$ was considered as statistically significant.

\section{Results}

\section{Population characteristics}

A total of 2,281 patients (1,420 males and 861 females) were recruited in the study, and $59.73 \pm 12.46$ years in all subjects, which 60.54 \pm 11.98 years in males and $58.38 \pm 13.11$ years in females. These subjects included 1,578 colorectal cancer patients and 703 controls. There were $1,529(96.89 \%)$ patients with adenocarcinomas, $40(2.53 \%)$ with mucinous carcinoma, and $9(0.57 \%)$ with signet ring cell carcinoma and others. There were 15(0.95\%), 1,480(93.79\%) and 61(3.87\%) patients with well, moderately and poorly differentiated tumors, respectively. And there were 22(1.39\%) patients were unknown. There were 40(2.53\%), 141(8.94\%), 827(52.41\%) and $431(27.31 \%)$ patients with T1, T2, T3 and T4 tumors according to depth of invasion, respectively. And there were $139(8.81 \%)$ patients were unknown. There were $604(38.28 \%)$ patients with stages I/II, $541(34.28 \%)$ with stage III, and $405(25.67 \%)$ with stage IV according to TNM classification of malignant tumors. The clinical characteristics of the subjects are presented in Table 1. 
Table 1

Baseline characteristics of subjects.

\begin{tabular}{|c|c|c|c|}
\hline & Colorectal cancer $(n=1578)$ & Controls $(n=703)$ & $P$ value \\
\hline Gender (male) & & & 0.044 \\
\hline Male, n (\%) & 1004(63.62) & $416(59.17)$ & \\
\hline Female, n (\%) & $574(36.38)$ & $287(40.83)$ & \\
\hline Age, mean \pm SD & $60.80 \pm 11.36(20-93)$ & $57.32 \pm 14.34(4-97)$ & $<0.001$ \\
\hline$\leq 60, \mathrm{n}(\%)$ & 754(47.78) & $399(56.76)$ & \\
\hline$>60, \mathrm{n}(\%)$ & $824(52.22)$ & $304(43.24)$ & \\
\hline \multicolumn{4}{|l|}{ Histology type } \\
\hline Adenocarcinoma, n (\%) & 1529(96.89) & & \\
\hline Mucinous carcinoma, n (\%) & $40(2.53)$ & & \\
\hline Signet ring cell carcinoma and others, $\mathrm{n}(\%)$ & $9(0.57)$ & & \\
\hline \multicolumn{4}{|l|}{ Differentiation } \\
\hline Well, n (\%) & $15(0.95)$ & & \\
\hline Moderate, n (\%) & 1480(93.79) & & \\
\hline Poor, n (\%) & $61(3.87)$ & & \\
\hline Unknown, n (\%) & 22(1.39) & & \\
\hline \multicolumn{4}{|l|}{ Depth of invasion } \\
\hline $\mathrm{T} 1, \mathrm{n}(\%)$ & $40(2.53)$ & & \\
\hline $\mathrm{T} 2, \mathrm{n}(\%)$ & $141(8.94)$ & & \\
\hline T3, n (\%) & $827(52.41)$ & & \\
\hline $\mathrm{T} 4, \mathrm{n}(\%)$ & $431(27.31)$ & & \\
\hline Unknown, n (\%) & $139(8.81)$ & & \\
\hline \multicolumn{4}{|l|}{ TNM stage } \\
\hline I/II, n (\%) & $604(38.28)$ & & \\
\hline III, n (\%) & $541(34.28)$ & & \\
\hline IV, n (\%) & $405(25.67)$ & & \\
\hline Unknown, n (\%) & $28(1.77)$ & & \\
\hline
\end{tabular}

Comparison of the CEA, CA24-2 and CA19-9 concentrations in the colorectal cancer group and control group

Comparison of the CEA, CA24-2 and CA19-9 concentrations among groups, showed that the levels of CEA $(P<0.001), C A 24-2(P<0.001)$ and CA19-9 $(P<0.001)$ in colorectal cancer group was significantly higher than control group. There were no significant differences in serum CEA $(P=0.741), C A 24-2(P=0.625)$ and CA19-9 $(P=0.738)$ levels among adenocarcinoma group, mucinous carcinoma group and signet ring cell carcinoma and others. Detailed data are presented in Table 2. 
Table 2

Different levels of tumor markers were observed among groups.

\begin{tabular}{|c|c|c|c|}
\hline & CEA & CA24-2 & CA19-9 \\
\hline Colorectal cancer $(n=1578)$ & $47.89 \pm 415.02$ & $31.01 \pm 166.83$ & $69.18 \pm 385.15$ \\
\hline controls $(n=703)$ & $3.63 \pm 14.05$ & $6.53 \pm 14.22$ & $34.42 \pm 437.64$ \\
\hline$P$ value & $<0.001$ & $<0.001$ & $<0.001$ \\
\hline \multicolumn{4}{|l|}{ Histology type } \\
\hline Adenocarcinoma $(n=1529)$ & $48.27 \pm 421.38$ & $30.86 \pm 167.41$ & $69.84 \pm 390.45$ \\
\hline Mucinous carcinoma $(n=40)$ & $26.91 \pm 84.59$ & $41.17 \pm 164.64$ & $55.51 \pm 156.47$ \\
\hline Signet ring cell carcinoma and others $(n=9)$ & $6.03 \pm 13.08$ & $11.00 \pm 18.70$ & $17.95 \pm 20.10$ \\
\hline$P$ value & 0.908 & 0.870 & 0.899 \\
\hline \multicolumn{4}{|l|}{ Differentiation } \\
\hline Well $(n=15)$ & $54.92 \pm 134.56$ & $8.77 \pm 13.35$ & $54.81 \pm 100.31$ \\
\hline Moderate $(n=1480)$ & $47.88 \pm 427.68$ & $27.79 \pm 144.43$ & $65.05 \pm 376.43$ \\
\hline Poor $(n=61)$ & $39.64 \pm 106.33$ & $102.98 \pm 441.46$ & $157.79 \pm 612.57$ \\
\hline$P$ value & 0.998 & 0.005 & 0.298 \\
\hline \multicolumn{4}{|l|}{ Depth of invasion } \\
\hline T1 $(n=40)$ & $2.32 \pm 1.72$ & $5.61 \pm 4.49$ & $13.71 \pm 13.48$ \\
\hline T2 $(n=141)$ & $12.58 \pm 61.26$ & $6.26 \pm 11.68$ & $18.75 \pm 42.25$ \\
\hline T3 (n = 827) & $17.48 \pm 82.80$ & $12.99 \pm 69.22$ & $32.37 \pm 189.71$ \\
\hline T4 $(n=431)$ & $46.64 \pm 161.87$ & $46.45 \pm 181.76$ & $86.75 \pm 247.96$ \\
\hline$P$ value & $<0.001$ & $<0.001$ & $<0.001$ \\
\hline \multicolumn{4}{|l|}{ TNM stage } \\
\hline $\mathrm{I} / \mathrm{II}(\mathrm{n}=604)$ & $5.57 \pm 20.39$ & $5.74 \pm 13.04$ & $15.26 \pm 39.66$ \\
\hline III $(n=541)$ & $12.03 \pm 62.02$ & $10.28 \pm 37.73$ & $22.52 \pm 65.27$ \\
\hline IV $(n=405)$ & $159.33 \pm 805.78$ & $98.09 \pm 316.85$ & $214.97 \pm 736.23$ \\
\hline$P$ value & $<0.001$ & $<0.001$ & $<0.001$ \\
\hline
\end{tabular}

Specificity and sensitivity of individual tumor marker and combination of these markers for colorectal cancer

In this study, tumor markers were detected in colorectal cancer patients and controls. Individual sensitivity sorted in descending order, the tumor markers were CEA (28.71\%) > CA19-9 (16.92\%) > CA24-2 (12.74\%). The combination of tumor markers can improve the sensitivity, and the combination of CEA + CA19-9 + CA24-2 ranked the highest in sensitivity index (34.92\%) in colorectal cancer. The individual specificity of tumor markers were CA24-2 (96.44\%) > CA19-9 (92.46\%) > CEA (92.18\%). Specificity can be improved by combining with tumor markers, and the combination of CEA + CA19-9 + CA24-2 ranked the highest in specificity index (98.86\%) in colorectal cancer (Table 3). 
Table 3

Sensitivity and specificity (\%) of tumor markers for colorectal cancer.

\begin{tabular}{|llll|}
\hline Tumor markers & Sensitivity $(\%)$ & Specificity $(\%)$ & Youden's index \\
\hline CEA & $28.71(453 / 1578)$ & $92.18(648 / 703)$ & 0.21 \\
\hline CA24-2 & $12.74(201 / 1578)$ & $96.44(678 / 703)$ & 0.09 \\
\hline CA19-9 & $16.92(267 / 1578)$ & $92.46(650 / 703)$ & 0.09 \\
\hline CEA+ CA24-2 & $32.19(508 / 1578)$ & $98.15(690 / 703)$ & 0.30 \\
\hline CEA+ CA19-9 & $34.16(539 / 1578)$ & $98.15(690 / 703)$ & 0.32 \\
\hline CA24-2+ CA19-9 & $19.84(313 / 1578)$ & $97.87(688 / 703)$ & 0.18 \\
\hline CEA + CA24-2+ CA19-9 & $34.92(551 / 1578)$ & $98.86(695 / 703)$ & 0.34 \\
\hline
\end{tabular}

\section{Comparison on ROC curves of CEA, CA24-2 and CA19-9}

Comparison the accuracies when using CEA, CA24-2 and CA19-9 in diagnosis of colorectal cancer by calculating area under the ROC curve (AUROC) (Fig. 1). In colorectal cancer, the AUC of CEA, CA24-2 and CA19-9 were 0.637, 0.580 and 0.565, respectively. Detailed data are presented in Table 4 and Fig. 1. Logistic regression analysis showed that the prediction equation excluding the risk of colorectal cancer was. Probability (normal) $=\operatorname{Exp}(-5.47-0.28 \mathrm{CEA}-0.11 \mathrm{CA} 242+0.001$ CA199)/ $(1+$ Exp $(-5.47-0.28 \mathrm{CEA}-0.11 \mathrm{CA} 242+0.001$ CA199)). When the calculated value was close to 0 , the risk of colorectal cancer was higher. The risk of colorectal cancer was lower when the value approached 1 .

Table 4

Areas under the ROC curve and predictive value of four tumor markers for colorectal cancer.

\begin{tabular}{|llll|}
\hline & CEA & CA24-2 & CA19-9 \\
\hline $95 \% \mathrm{Cl}$ & $0.614-0.650$ & $0.556-0.604$ & $0.540-0.590$ \\
\hline P value & $<0.001$ & $<0.001$ & $<0.001$ \\
\hline AUC & 0.637 & 0.580 & 0.565 \\
\hline Cut-off point & 2.495 & 6.140 & 19.63 \\
\hline Specificity (\%) & 50.0 & 39.3 & 30.0 \\
\hline Sensitivity (\%) & 71.7 & 74.1 & 81.2 \\
\hline 95\% Cl, 95\% confidence interval; AUC, area under the ROC curve. \\
\hline
\end{tabular}

The association between KRAS/NRAS, PIK3CA and BRAF genes status and clinical characteristics

Among colorectal cancer patients, 458 were tested for KRAS/NRAS mutations, 431 for BRAF mutations, and 146 for PIK3CA mutations. Among them, 216 patients (47.2\%) with KRAS/NRAS mutations, 11 patients $(2.6 \%)$ with BRAF mutations and 14 patients $(9.6 \%)$ with PIK3CA mutations. There were no significant differences in age, gender, histology type, differentiation, depth of invasion and TNM stage between KRAS/NRAS (+) group and KRAS/NRAS (-) group, BRAF (+) group and BRAF (-) group, PIK3CA (+) group and PIK3CA (-) group, respectively (Table 5). 
Table 5

Analysis of the relationship between KRAS/NRAS, PIK3CA and BRAF genes status and clinical characteristics.

\begin{tabular}{|c|c|c|c|c|c|c|c|c|c|}
\hline \multirow[b]{2}{*}{ Characteristic } & \multicolumn{3}{|c|}{ KRAS/NRAS mutation } & \multicolumn{3}{|c|}{ PIK3CA mutation } & \multicolumn{3}{|c|}{ BRAF mutation } \\
\hline & $\begin{array}{l}+(n= \\
216)\end{array}$ & $\begin{array}{l}-(n= \\
242)\end{array}$ & $P$ value & $+(n=14)$ & $\begin{array}{l}-(n= \\
132)\end{array}$ & $P$ value & $\begin{array}{l}+(n= \\
11)\end{array}$ & $\begin{array}{l}-(n= \\
420)\end{array}$ & $P$ value \\
\hline Age & & & $\begin{array}{l}0.454\left(x^{2}\right. \\
=0.576)\end{array}$ & & & $\begin{array}{l}0.173\left(x^{2}\right. \\
=2.017)\end{array}$ & & & $\begin{array}{l}0.762\left(x^{2}\right. \\
=0.251)\end{array}$ \\
\hline$\leq 60$ & $95(44.0)$ & 115(47.5) & & $4(28.6)$ & $64(48.5)$ & & $6(54.5)$ & 197(46.9) & \\
\hline$₫ 60$ & 121(56.0) & $127(52.5)$ & & $10(71.4)$ & $68(51.5)$ & & $5(45.5)$ & 223(53.1) & \\
\hline Gender & & & $\begin{array}{l}0.277\left(x^{2}\right. \\
=1.362)\end{array}$ & & & $\begin{array}{l}0.381\left(x^{2}\right. \\
=1.257)\end{array}$ & & & $\begin{array}{l}0.537\left(x^{2}\right. \\
=0.037)\end{array}$ \\
\hline Male & $137(63.4)$ & 166(68.6) & & $7(50.0)$ & $86(65.2)$ & & $7(63.6)$ & $279(66.4)$ & \\
\hline Female & $79(36.6)$ & $76(31.4)$ & & $7(50.0)$ & $46(34.8)$ & & $4(36.4)$ & 141(33.6) & \\
\hline Histology type & & & $\begin{array}{l}0.255\left(x^{2}\right. \\
=2.729)\end{array}$ & & & $\begin{array}{l}0.760\left(x^{2}\right. \\
=0.549)\end{array}$ & & & $\begin{array}{l}0.250\left(x^{2}\right. \\
=2.776)\end{array}$ \\
\hline Adenocarcinoma & 212(98.1) & 234(96.7) & & $14(100.0)$ & $127(96.2)$ & & $10(90.9)$ & $409(97.4)$ & \\
\hline $\begin{array}{l}\text { Mucinous } \\
\text { carcinoma }\end{array}$ & $4(0.9)$ & $5(2.1)$ & & $0(9.5)$ & $4(3.0)$ & & $1(9.1)$ & $8(1.9)$ & \\
\hline $\begin{array}{l}\text { Signet ring cell } \\
\text { carcinoma and } \\
\text { others }\end{array}$ & $0(0)$ & $3(1.2)$ & & $0(0)$ & $1(0.8)$ & & $0(0)$ & $3(0.7)$ & \\
\hline Differentiation & & & $\begin{array}{l}0.122\left(\chi^{2}\right. \\
=5.803)\end{array}$ & & & $\begin{array}{l}0.202\left(x^{2}\right. \\
=4.616)\end{array}$ & & & $\begin{array}{l}0.873\left(\chi^{2}\right. \\
=0.699)\end{array}$ \\
\hline Well & $5(2.3)$ & $0(0)$ & & $1(7.1)$ & $1(0.8)$ & & $0(0)$ & $5(1.2)$ & \\
\hline Moderate & $200(92.6)$ & $228(94.2)$ & & $13(92.9)$ & 123(93.2) & & $10(90.9)$ & $393(93.6)$ & \\
\hline Poor & $9(4.2)$ & $12(5.0)$ & & $0(0)$ & $7(5.3)$ & & $1(9.1)$ & $19(4.5)$ & \\
\hline $\begin{array}{l}\text { Depth of } \\
\text { invasion }\end{array}$ & & & $\begin{array}{l}0.398\left(x^{2}\right. \\
=5.152)\end{array}$ & & & $\begin{array}{l}0.492\left(x^{2}\right. \\
=2.408)\end{array}$ & & & $\begin{array}{l}0.693\left(x^{2}\right. \\
=3.047)\end{array}$ \\
\hline $\mathrm{T} 1$ & $2(0.9)$ & $7(2.9)$ & & $0(0)$ & $0(0)$ & & $0(0)$ & $7(1.7)$ & \\
\hline T2 & $17(7.9)$ & $17(7.0)$ & & $1(7.1)$ & $5(3.8)$ & & $2(18.2)$ & $30(7.1)$ & \\
\hline T3 & 127(58.8) & 128(52.9) & & $9(64.3)$ & $71(53.8)$ & & $5(45.5)$ & 231(55.0) & \\
\hline $\mathrm{T} 4$ & $51(23.6)$ & $65(26.9)$ & & $4(28.6)$ & $39(29.5)$ & & $2(18.2)$ & $107(25.5)$ & \\
\hline TNM stage & & & $\begin{array}{l}0.176\left(x^{2}\right. \\
=4.949)\end{array}$ & & & $\begin{array}{l}0.150\left(x^{2}\right. \\
=5.321)\end{array}$ & & & $\begin{array}{l}0.382\left(x^{2}\right. \\
=3.064)\end{array}$ \\
\hline $\mathrm{I} / \mathrm{II}$ & $72(33.3)$ & $78(32.2)$ & & $4(28.6)$ & $22(16.7)$ & & $6(54.5)$ & 129(30.7) & \\
\hline III & $70(32.4)$ & $86(35.5)$ & & $8(57.1)$ & 49(37.1) & & 2(18.2) & 142(33.8) & \\
\hline IV & 73(33.8) & $71(29.3)$ & & $2(14.3)$ & $59(44.7)$ & & $3(27.3)$ & 141(33.6) & \\
\hline
\end{tabular}

\section{Discussion}

Colorectal cancer is the most common malignant tumor in human digestive tract[18,19]. With the development of researches in recent years, tumor markers have been used in the early diagnosis of various tumors[20-23]. Tumor markers refer to substances synthesized and secreted by tumor cells and released into blood, cells and body fluids, which can reflect the occurrence and development of tumor[20,23,24]. CEA is a proteoglycan complex produced by colorectal cancer, which is widely found in cancers of the digestive 
system[25, 26]. CA24-2 is a kind of salivary acidified spingolipid glycosylated chain antigen, which is expressed in human pancreatic duct cells and colonic mucosal epithelial cells. It is mainly used in clinical detection of pancreatic cancer and colorectal cancer[27]. CA19-9 is a specific tumor marker for adenocarcinoma, but it lacks organ specificity and can be expressed in various malignancies such as pancreatic cancer, gastric cancer and colorectal cancer[28, 29]. The levels of CEA, CA19-9 and CA24-2 are significant for the diagnosis and treatment of colorectal cancer.

In this study, three common serum markers (CEA, CA19-9 and CA24-2) of colorectal cancer were evaluated separately and jointly evaluated. The serum concentrations of CEA, CA24-2 and CA19-9 in colorectal cancer patients were significantly different from those in the control group, suggesting that CEA, CA24-2 and CA19-9 may be of important value in the diagnosis of colorectal cancer. By analyzing the relationship between serum CEA, CA19-9 and CA24-2 and clinical TNM staging, it was found that the concentrations of CEA, CA19-9 and CA24-2 changed with the degree of infiltration and TNM staging, showing a positive correlation. The concentration of CA24-2 was negatively correlated with the degree of tumor differentiation. The differences between CEA, CA19-9 concentration and tumor differentiation degree was not statistically significant. This is consistent with other studies[30, 31]. There are also inconsistencies with some studies[29, 32].

The sensitivity, specificity and ROC curve of these tumor markers were analyzed. The sensitivities of three individual markers were lower than $30 \%$, which individual sensitivity of the tumor markers sorted in descending order were CEA > CA19-9 > CA24-2. The specificities of three individual markers were more than $92 \%$, and the specificity sorted in descending order were CA24-2 > CA19-9 > CEA. The sensitivities and specificities of tumor markers can be improved by combination of these markers. Although the combined detection of CEA, CA24-2 and CA19-9 can improve the sensitivities of diagnosis, there were no significant differences compared with the single detection of CEA, CA24-2 and CA19-9.

There were no significant differences in age, gender, histology type, differentiation, depth of invasion and TNM stage between KRAS/NRAS (+) group and KRAS/NRAS (-) group, BRAF (+) group and BRAF (-) group, PIK3CA (+) group and PIK3CA (-) group, respectively. It showed that mutations in KRAS/NRAS, BRAF and PIK3CA genes were not associated with pathological status of colorectal cancer. There are different domestic and foreign studies on the relationship between KRAS/NRAS, BRAF and PIK3CA gene mutation status and pathological characteristics in colorectal cancer, which may be related to the detection method, sample size and regional differences[33-35].

In recent years, many new biomarkers of colorectal cancer have been found due to the research progress in molecular subtypes, DNA methylation, microRNA biogenesis and their role in colorectal cancer. These biomarkers can be used for early diagnosis, individualized treatment and prognosis of colorectal cancer[36]. For example, hsa-mir-183-5p and hsa-mir-21-5p[37], hsa-mir-30a[38], hsa-mir-96[39], hsa-mir-21[40] for diagnosis of colorectal cancer. And hsa-mir-143 and hsa-mir-145 for prognosis of colorectal cancer[41]. We need to find more specific and sensitive markers for early diagnosis, therapeutic evaluation and prognosis of colorectal cancer.

\section{Conclusions}

There is no single serum marker sensitive enough for diagnosis and screening of colorectal cancer. Combined with tumor markers can be used to improve the clinical efficacy for colorectal cancer diagnosis. New specific and sensitive markers for early diagnosis, therapeutic evaluation and prognosis of colorectal cancer also needed.

\section{Declarations}

Ethics approval and consent to participate

The study was approved by the Ethics Committee of Medicine, Meizhou People's Hospital (Huangtang Hospital), Meizhou Academy of Medical Sciences, Meizhou Hospital Affiliated to Sun Yat-sen University.

Consent for publication

Not applicable.

Availability of data and materials 
The data used to support the findings of this study are available from the corresponding author upon request.

Competing interests

The authors declare that they have no competing interests.

Funding

This study was supported by Key Scientific and Technological Project of Meizhou People's Hospital, (Grant No.: MPHKSTP-20180101 to Dr. Zhixiong Zhong).

Authors' contributions

Zhixiong Zhong and Heming Wu designed the study. Hui Rao, Qingyan Huang, Zhikang Yu and Qunji Zhang performed the experiments. Heming Wu and Hui Rao collected clinical data. Heming Wu and Hui Rao analyzed the data. Heming Wu prepared the manuscript. All authors were responsible for critical revisions, and all authors read and approved the final version of this work.

Acknowledgments

The author would like to thank other colleagues whom were not listed in the authorship of Center for Precision Medicine, Meizhou People's Hospital (Huangtang Hospital), Meizhou Academy of Medical Sciences, Meizhou Hospital Affiliated to Sun Yat-sen University for their helpful comments on the manuscript.

\section{References}

1. Weitz J, Koch M, Debus J, Hohler T, Galle PR, Buchler MW: Colorectal cancer. Lancet 2005, 365:153-165.

2. Cunningham D, Atkin W, Lenz HJ, Lynch HT, Minsky B, Nordlinger B, Starling N: Colorectal cancer. Lancet 2010, 375:1030-1047.

3. Haggar F, Boushey R: Colorectal Cancer Epidemiology: Incidence, Mortality, Survival, and Risk Factors. Clin Colon Rectal Surg, 22:191-197.

4. Siegel RL, Miller KD, Fedewa SA, Ahnen DJ, Meester RGS, Barzi A, Jemal A: Colorectal cancer statistics, 2017. CA Cancer J Clin 2017, 67:177-193.

5. Cook AD, Single R, McCahill LE: Surgical resection of primary tumors in patients who present with stage IV colorectal cancer: an analysis of surveillance, epidemiology, and end results data, 1988 to 2000. Ann Surg Oncol 2005, 12:637-645.

6. Qiu M, Hu J, Yang D, Cosgrove DP, Xu R: Pattern of distant metastases in colorectal cancer: a SEER based study. Oncotarget, 6.

7. Patel NN, Shah PR, Wilson E, Haray PN: An unexpected supraclavicular swelling. World J Surg Oncol 2007, 5:90.

8. Davies JM, Goldberg RM: Treatment of metastatic colorectal cancer. Semin Oncol 2011, 38:552-560.

9. Ganapathy-Kanniappan S, Geschwind JF: Tumor glycolysis as a target for cancer therapy: progress and prospects. Mol Cancer 2013, 12:152.

10. Bates SE: Clinical applications of serum tumor markers. Ann Intern Med 1991, 115:623-638.

11. Perkins GL, Slater ED, Sanders GK, Prichard JG: Serum tumor markers. Am Fam Physician 2003, 68:1075-1082.

12. Plebani M, De Paoli M, Basso D, Roveroni G, Giacomini A, Galeotti F, Corsini A: Serum tumor markers in colorectal cancer staging, grading, and follow-up. J Surg Oncol 1996, 62:239-244.

13. Jing JX, Wang Y, Xu XQ, Sun T, Tian BG, Du LL, Zhao XW, Han CZ: Tumor markers for diagnosis, monitoring of recurrence and prognosis in patients with upper gastrointestinal tract cancer. Asian Pac J Cancer Prev 2014, 15:10267-10272.

14. Nakayama T, Watanabe M, Teramoto T, Kitajima M: Prognostic values of serum CA19-9 and CEA levels for colorectal cancer. Oncol Rep 1997, 4:819-822.

15. Tokunaga R, Sakamoto Y, Nakagawa S, Yoshida N, Baba H: The utility of tumor marker combination, including serum P53 antibody, in colorectal cancer treatment. Surg Today 2017, 47:636-642.

16. Ohtsuka T, Nakafusa Y, Sato S, Kitajima Y, Tanaka M, Miyazaki K: Different roles of tumor marker monitoring after curative resections of gastric and colorectal cancers. Dig Dis Sci 2008, 53:1537-1543.

17. Kazama S, Watanabe T: Diagnosis of colorectal cancer by measurement of tumor markers. Nihon Rinsho 2014, 72:71-76. 
18. Haraldsdottir S, Einarsdottir HM, Smaradottir A, Gunnlaugsson A, Halfdanarson TR: Colorectal cancer - review. Laeknabladid 2014, 100:75-82.

19. Wang J, Liang W, Wang X, Min G, Chen W, Wang H, Yao N, Wang J: The value of biomarkers in colorectal cancer: Protocol for an overview and a secondary analysis of systematic reviews of diagnostic test accuracy. Medicine (Baltimore) 2019, 98:e16034.

20. Diamandis EP: Towards identification of true cancer biomarkers. BMC Med 2014, 12:156.

21. Schiffman JD, Fisher PG, Gibbs P: Early detection of cancer: past, present, and future. Am Soc Clin Oncol Educ Book 2015:57-65.

22. Shen $\mathrm{M}$, Wang H, Wei K, Zhang J, You C: Five common tumor biomarkers and CEA for diagnosing early gastric cancer: A protocol for a network meta-analysis of diagnostic test accuracy. Medicine (Baltimore) 2018, 97:e0577.

23. Chan KC: Scanning for cancer genomic changes in plasma: toward an era of personalized blood-based tumor markers. Clin Chem 2013, 59:1553-1555.

24. Suresh MR: Classification of tumor markers. Anticancer Res 1996, 16:2273-2277.

25. Duffy MJ, Lamerz R, Haglund C, Nicolini A, Kalousová M, Holubec L, Sturgeon C: Tumor markers in colorectal cancer, gastric cancer and gastrointestinal stromal cancers: European group on tumor markers 2014 guidelines update. Int J Cancer 2014, 134:25132522.

26. Campos-da-Paz M, Dórea JG, Galdino AS, Lacava ZGM, de Fatima Menezes Almeida Santos M: Carcinoembryonic Antigen (CEA) and Hepatic Metastasis in Colorectal Cancer: Update on Biomarker for Clinical and Biotechnological Approaches. Recent Pat Biotechnol 2018, 12:269-279.

27. Zhang XC, Zhang JH, Wang RF, Fan Y, Fu ZL, Yan P, Zhao GY, Bai YX: Diagnostic value of (18)F-FDG PET/CT and tumor markers (CEA, CA19-9, CA24-2) in recurrence and metastasis of postoperative colorectal moderately differentiated adenocarcinoma. Beijing Da Xue Xue Bao Yi Xue Ban 2019, 51:1071-1077.

28. Komori A, Taniguchi H, Hamauchi S, Masuishi T, Kito Y, Narita Y, Tsushima T, Ishihara M, Todaka A, Tanaka T, et al: Serum CA19-9 Response Is an Early Predictive Marker of Efficacy of Regorafenib in Refractory Metastatic Colorectal Cancer. Oncology 2017, 93:329-335.

29. Gao Y, Wang J, Zhou Y, Sheng S, Qian SY, Huo X: Evaluation of Serum CEA, CA19-9, CA72-4, CA125 and Ferritin as Diagnostic Markers and Factors of Clinical Parameters for Colorectal Cancer. Sci Rep 2018, 8:2732.

30. Wang J, Wang X, Yu F, Chen J, Zhao S, Zhang D, Yu Y, Liu X, Tang H, Peng Z: Combined detection of preoperative serum CEA, CA199 and CA242 improve prognostic prediction of surgically treated colorectal cancer patients. Int J Clin Exp Pathol 2015, 8:1485314863.

31. Ning S, Wei W, Li J, Hou B, Zhong J, Xie Y, Liu H, Mo X, Chen J, Zhang L: Clinical significance and diagnostic capacity of serum TK1, CEA, CA 19-9 and CA 72-4 levels in gastric and colorectal cancer patients. J Cancer 2018, 9:494-501.

32. Attallah AM, El-Far M, Ibrahim AR, El-Desouky MA: Clinical value of a diagnostic score for colon cancer based on serum CEA, CA199, cytokeratin-1 and mucin-1. 2018, 75:122-127.

33. Guo F, Gong H, Zhao H, Chen J, Zhang Y, Zhang L, Shi X, Zhang A, Jin H, Zhang J, He Y: Mutation status and prognostic values of KRAS, NRAS, BRAF and PIK3CA in 353 Chinese colorectal cancer patients. Sci Rep 2018, 8:6076.

34. Reggiani Bonetti L, Barresi V: Clinical Impact and Prognostic Role of KRAS/BRAF/PIK3CA Mutations in Stage I Colorectal Cancer. 2018, 2018:2959801.

35. Zhang J, Zheng J, Yang Y, Lu J, Gao J, Lu T, Sun J, Jiang H, Zhu Y, Zheng Y, et al: Molecular spectrum of KRAS, NRAS, BRAF and PIK3CA mutations in Chinese colorectal cancer patients: analysis of 1,110 cases. Sci Rep 2015, 5:18678.

36. Yiu AJ, Yiu CY: Biomarkers in Colorectal Cancer. Anticancer Res 2016, 36:1093-1102.

37. Falzone L, Scola L, Zanghì A, Biondi A, Di Cataldo A, Libra M, Candido S: Integrated analysis of colorectal cancer microRNA datasets: identification of microRNAs associated with tumor development. Aging (Albany NY) 2018, 10:1000-1014.

38. Jin H, Shi X, Zhao Y, Peng M, Kong Y, Qin D, Lv X: MicroRNA-30a Mediates Cell Migration and Invasion by Targeting Metadherin in Colorectal Cancer. Technol Cancer Res Treat 2018, 17:1533033818758108.

39. Iseki Y, Shibutani M, Maeda K, Nagahara H, Fukuoka T, Matsutani S, Hirakawa K, Ohira M: MicroRNA-96 Promotes Tumor Invasion in Colorectal Cancer via RECK. Anticancer Res 2018, 38:2031-2035.

40. Tsukamoto M, linuma H, Yagi T, Matsuda K, Hashiguchi Y: Circulating Exosomal MicroRNA-21 as a Biomarker in Each Tumor Stage of Colorectal Cancer. Oncology 2017, 92:360-370. 
41. Li C, Yan G, Yin L, Liu T, Li C, Wang L: Prognostic roles of microRNA 143 and microRNA 145 in colorectal cancer: A meta-analysis. Int J Biol Markers 2019, 34:6-14.

\section{Abbreviations}

CEA, carcinoembryonic antigen;

CA24-2, carbohydrate antigen 24-2;

CA19-9, carbohydrate antigen 19-9;

$\mathrm{ADK}$, adenocarcinomas;

TNM, TNM classification of malignant tumors;

AUC, area under the ROC curve.

\section{Figures}




\section{ROC Curve}

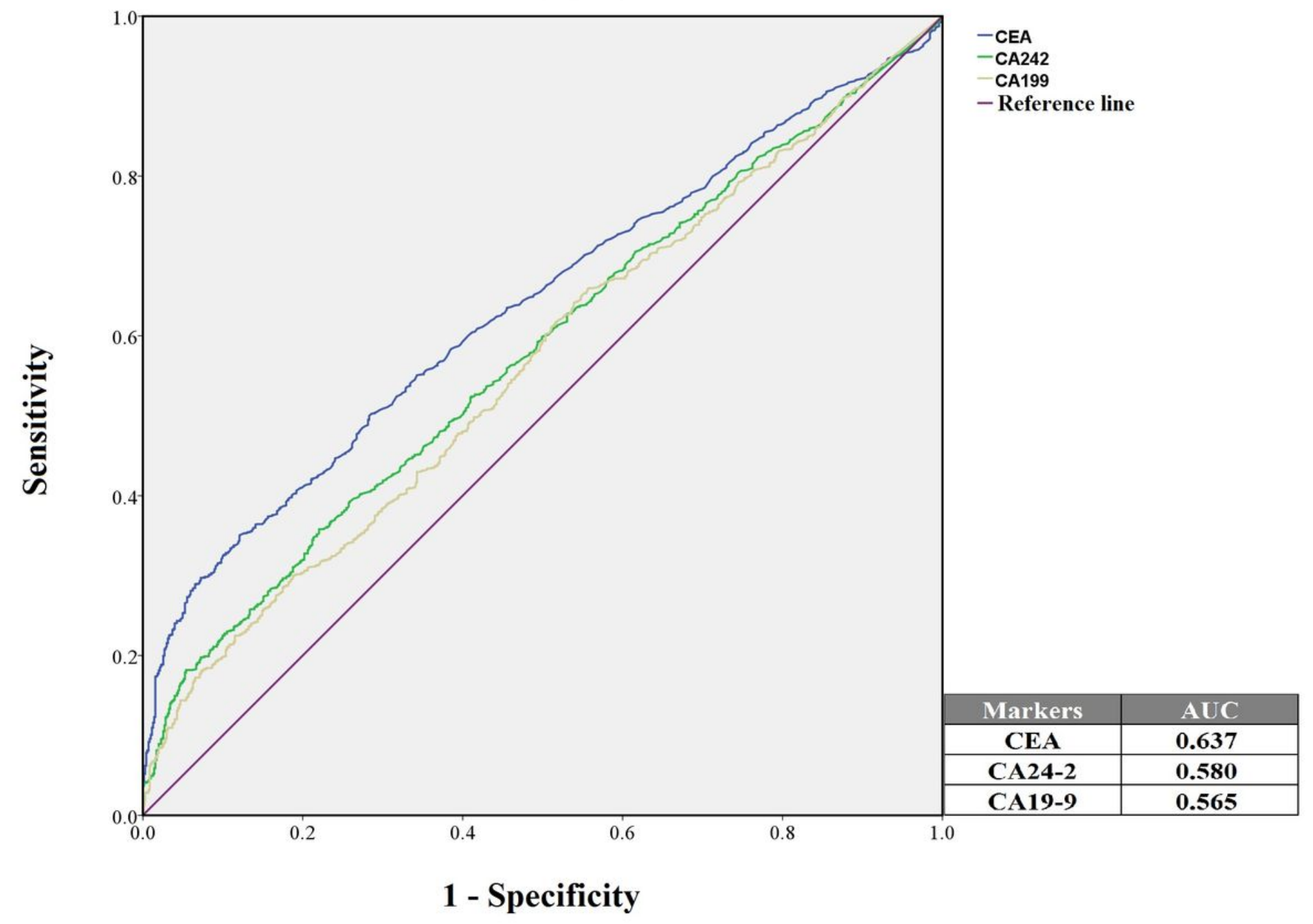

Figure 1

ROC Curves of CEA, CA24-2 and CA19-9 for colorectal cancer 